\title{
Diseño e implementación de un sistema de control a lazo cerrado PID para manipular la temperatura en el proceso de termoformado
}

\section{Design and implementation of a PID closed-loop control system to manipulate the temperature in the thermoforming process}

ROSALES-DAVALOS, Jaime $\dagger^{*}$, GIL-ANTONIO, Leopoldo, MASTACHE-MASTACHE, Jorge Edmundo y LÓPEZ-RAMÍREZ, Roberto

Tecnológico de Estudios Superiores de Jocotitlán, Carretera Toluca-Atlacomulco KM 44.8, Ejido de San Juan y San Agustín Jocotitlán, 50700 Jocotitlán, Estado de México, México.

ID $1^{\mathrm{er}}$ Autor: Jaime, Rosales-Davalos / ORC ID: 0000-0002-9059-6093, Researcher ID Thomson: MVQ9OX-GXKHUL, CVU CONACYT ID: 812961

ID $1{ }^{\text {er }}$ Coautor: Leopoldo, Gil-Antonio / ORC ID: 0000-0002-7445-9426

ID $2^{\text {do }}$ Coautor: Jorge Edmundo, Mastache-Mastache / ORC ID: 0000-0001-6104-6764, Researcher ID Thomson: H-11872018, CVU CONACYT ID: 544943

ID $3{ }^{\text {er }}$ Coautor: Roberto, López-Ramírez / ORC ID: 0000-0001-8341-3684, CVU CONACYT ID: 233228, SNI CONACYT ID: 63850

\section{Resumen}

El propósito de este trabajo radicó en el diseño e implementación de un sistema de control de temperatura a lazo cerrado (SCTLC), para controlar la transferencia temperatura en el molde de un sistema de termoprensado, donde se fabrican bloques a partir de los envases multicapa de posconsumó. Se caracterizó la planta (resistencia eléctrica), con los niveles de tensión de corriente alterna $(30 \mathrm{~V}, 60 \mathrm{~V}, 90 \mathrm{~V}$ y $120 \mathrm{~V})$ en un tiempo de $60 \mathrm{~min}$. Cada uno de los niveles de voltaje se consideró como temperatura inicial la del ambiente. Posteriormente se determinó el modelo de la planta utilizando el software Matlab ${ }^{\circledR}$ y un método analítico con los datos de la curva característica de la planta a una tensión de $120 \mathrm{~V}_{\mathrm{AC}}$, obteniendo la función de transferencia de primer orden. Así mismo, se sintonizo el controlador PID implementado el método Ziegler \& Nichols y el software de Matlab $^{\circledR}$ y finalmente se acoplo la entrada de referencia, el control, la etapa de potencia, planta y la retroalimentación. Este proyecto contribuye a controlar y estabilizar la temperatura de la resistencia, implementada en el proceso de termoformado, para la elaboración de bloques utilizando envases multicapas.

Sistema de control a lazo cerrado, Temperatura, Bloques

\begin{abstract}
The purpose of this work lies in the design and implementation of a closed-circuit temperature control system (SCTLC), to control the temperature transfer in the mold of the thermoprepared system, where the blocks are manufactured from multilayer containers. post-consumer. The plant (electrical resistance) was characterized, with the $\mathrm{AC}$ voltage levels $(30 \mathrm{~V}$, $60 \mathrm{~V}, 90 \mathrm{~V}$ and $120 \mathrm{~V}$ ) in a time of $60 \mathrm{~min}$ each of the voltage levels, considering the initial temperature (ambient temperature), in function of the step signal, then, the plant model was determined using Matlab software and the analytical method with the data of the plant characteristic curve at a voltage of $120 \mathrm{Vac}$, obtaining the first-order transfer function, then The PID controller implemented the Ziegler \& Nichols method and the Matlab software were tuned and finally the reference input, the control, the power stage, the plant and the feedback were coupled. This project contributes to control and stabilize the resistance temperature, implemented in the thermoforming process, for the production of blocks using multilayer containers.
\end{abstract}

Closed loop control system, Temperature, Blocks

Citación: ROSALES-DAVALOS, Jaime, GIL-ANTONIO, Leopoldo, MASTACHE-MASTACHE, Jorge Edmundo y LÓPEZ-RAMÍREZ, Roberto. Diseño e implementación de un sistema de control a lazo cerrado PID para manipular la temperatura en el proceso de termoformado. Revista de Ingeniería Eléctrica. 2020. 4-12:24-29.

\footnotetext{
$\uparrow$ Investigador contribuyendo como primer autor.

*Correspondencia al Autor Correo Electrónico: jaime.rosales@tesjo.edu.mx)
} 


\section{Introducción}

Actualmente, los sistemas de control son importantes en el desarrollo, avance de la civilización y la tecnología; se encuentran en los sectores industriales, el control de calidad de los productos manufacturados, líneas de ensamble automático, control de máquinas y herramientas, tecnología espacial y sistemas de armas, control de computadoras, sistemas de transporte, sistemas de potencia, etc. (Kuo, 1996).

Desde entonces, se utilizan ampliamente en toda la industria, donde proporcionan un rendimiento satisfactorio y robusto para una variedad de procesos (Astróm \& Hagglund, 1995).

Los hornos eléctricos son utilizados en la industria y hogar en diferentes aplicaciones, habitualmente el calentamiento de los hornos eléctricos se realiza con resistencias eléctricas colocadas en la parte inferior y superior de la superficie del horno. Su operación del controlador on-off es inestable y el controlador PID es utilizado en tratamientos térmicos muy sensibles. (Grassi \& Tsakalis, 2000).

Además, se pretende que cada día los procesos sean más automatizados y con el mayor control posible que minimice los costos de operación y mantenimiento; pero a la vez, que brinde altos estándares de calidad. A su vez, dentro del control por realimentación, la estrategia basada en el algoritmo PID (Proporcional, Integral, Derivativo) (Alfaro , 2005).

La selección de los parámetros del controlador PID tiene un efecto significativo en el rendimiento del control (Yanmei, Yanzhu, \& Baoyu, 2011).

También reducir los tiempos de retardo o tiempo muerto, son una parte fundamental de la dinámica de muchos procesos industriales, siendo una limitante para obtener un sistema de control (Vargas , 2012).
Finalmente, las oscilaciones de la temperatura en un horno eléctrico con el valor de referencia dependen del sistema a lazo cerrado a implementar; es decir, con el sistema control onoff se presentan más oscilaciones con respecto al valor de referencia y es inestable, hay un error de compensación menor a la técnica anterior y las oscilaciones disminuyen con control P(proporcional), PI y PID el error desaparece (Güney, Temizkan, Tekin, Samuk, \& Cakir, 2020).

Por lo tanto, los sistemas de control se implementan para manipular las variables de control y de salida; que deben ser igual a los valores de referencia; los sistemas de control garantizan la estabilidad y son robustos a perturbaciones $\mathrm{y}$ errores en los modelos eficientes, que deben evitar comportamientos bruscos e irreales y fáciles de ejecutar y manipular en tiempo real. (Gutiérrez \& Iturralde, 2017).

El diseño e implementación de un sistema de control a lazo cerrado PID para manipular la temperatura en el proceso de termoformado disminuye las oscilaciones respecto al valor de referencia, mejora la estabilidad del sistema y tiempo muero del proceso. Así mismo, reduce el costo de mantenimiento y operación del sistema de control de temperatura on-off implementado en el proceso de termoformado para elaboración de bloque a partir de envases multicapas (Rosales, Enríquez , López, \& Mastache , 2019).

Anteriormente, se tenía un sistema de control de temperatura a lazo cerrado On-Off, presentaba tiempos muertos y era inestable; es decir, el valor de temperatura varia con respecto al valor de referencia de $10^{\circ} \mathrm{C}$ a $30^{\circ} \mathrm{C}$. Por eso, en este trabajo se reporta el diseño e implementación de la técnica de control PID para disminuir los componentes electrónicos y electromecánicos, también reducir el tiempo muerto y que sea estable el control de temperatura. 


\section{Descripción del método}

Se determinó la curva característica de la planta (resistencia eléctrica 200 Watts a 120 Volts); es decir, se graficó la temperatura en ${ }^{\circ} \mathrm{C}$ (grados Celsius) en función del tiempo (minutos), donde se suministró $30,60,90$ y $120 \mathrm{~V}_{\mathrm{AC}}$ (voltaje de corriente alterna) en un periodo de tiempo de 60 min, para cada uno de los niveles de tensión, considerando que la temperatura inicial es igual a la temperatura ambiente.

En la figura 1, se muestra el diagrama a bloques para la obtención de las curvas características de la planta, en otras palabras, el "termocuplas tipo K" obtiene la temperatura de la planta y la suministra al microcontrolador STM32F767ZI que la procesa. Por lo tanto, la salida del microcontrolador es un voltaje de $0 \mathrm{y}$ $7 \mathrm{~V}_{\mathrm{DC}}$ (volts de corriente directa). Dependiendo, del voltaje suministrado por el microcontrolador al circuito TCA785 se manipula el ángulo de disparo que determina la potencia entregada a la planta.

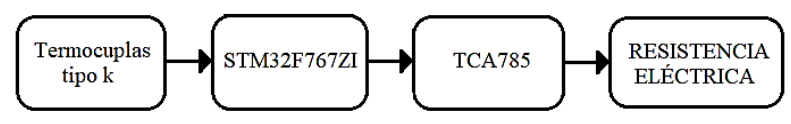

Figura 1 Diagrama de bloques para la caracterización de la planta

Fuente: Autoría propia

A continuación, de determino la función de transferencia de la planta con el software Matlab, considerando los datos de la curva característica de la resistencia eléctrica cuando se le aplico $120 \mathrm{~V}_{\mathrm{AC}}$ con un tiempo de 60 minutos, obteniendo una función de transferencia de orden uno, ver ecuación 1.

$G(s)=\frac{k}{T s+1}$

Después, se sintonizo el controlador PID, que se simulo con el software de Matlab, en otras palabras, se determinaron los valores de las constantes proporcional $\left(\mathrm{K}_{\mathrm{p}}\right)$, integral $\left(\mathrm{T}_{\mathrm{i}}\right)$ y derivativo $\left(\mathrm{T}_{\mathrm{d}}\right)$. Por otro lado, se implementó el sistema de control a lazo cerrado PID, ver figura 2 (Ogata, 1998).
La entrada de referencia, el comparador y el controlador PID se implementaron a través del microcontrolador "STM32F767ZI"; los circuitos de aislamiento y control de fase se utiliza el circuito TCA785 y un optotriac y el actuador está constituido por el tiristor (triac), la planta es la resistencia eléctrica y para obtener la señal de retroalimentación se utiliza el termocupla tipo $\mathrm{k}$ su función es captar temperatura de la planta y enviarla al elemento de comparación.

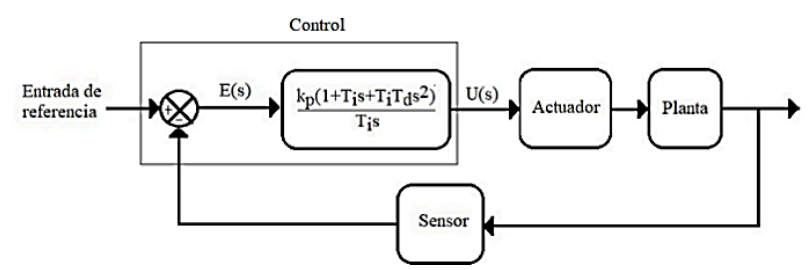

Figura 2 Diagrama de bloques de un sistema de control industrial

Fuente: (Ogata, 1998)

\section{Resultados}

El algoritmo para caracterizar la planta, en otras palabras, determinar la curva de característica de la resistencia eléctrica en función de señal escalón, ver figura 3.

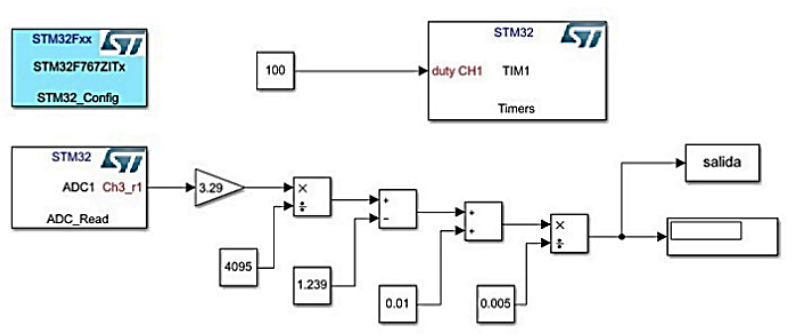

Figura 3 Programa en Simulink ${ }^{\circledR}$ para adquisición de datos

Fuente: Autoría propia

Las curvas características de la planta para los diferentes niveles de voltaje de 30,60, 90 y $120 \mathrm{~V}_{\mathrm{AC}}$, en un tiempo de $60 \mathrm{~min}$, se muestran en la figura 4 . Con un tiempo de prueba entre 0 y 500 segundos la temperatura se incrementa y posteriormente de 500 a 3600 segundos se mantiene constante con algunas oscilaciones. Para cada una de las curvas características, se observa que al aumentar potencia en la resistencia eléctrica aumenta la temperatura de estabilización. 


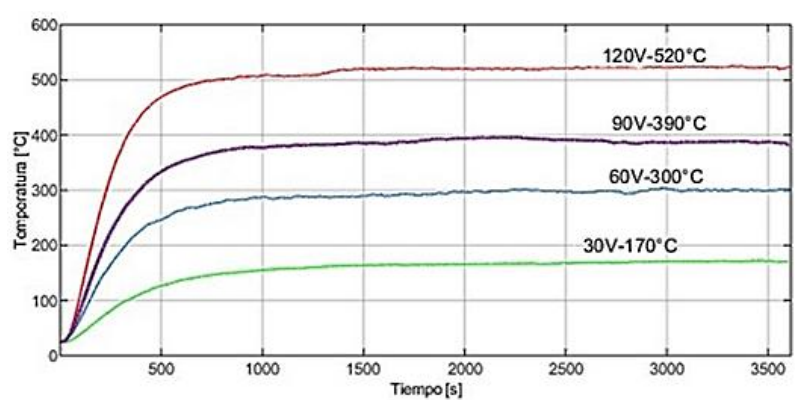

Figura 4 Curvas características de la planta Fuente: Autoría propia

Posteriormente, se calcula la función de transferencia de la planta utilizando la herramienta "Plant Identification" del software Matlab $^{\circledR}$, utilizando los datos de la curva característica de $120 \mathrm{Vac}$ y $520{ }^{\circ} \mathrm{C}$ y la señal escalón de 120 Volts, donde $k=4.339, T=$ 227.9 , finalmente sustituyendo en la ecuación 1 se obtiene la ecuación (2).

$G(s)=\frac{4.339}{227.9 s+1}$

En la figura 5, se muestran las gráficas de respuesta de la función de transferencia de la planta simulado, así mismo se presenta la respuesta de la planta considerando las condiciones de la simulación de la función de transferencia real, se observa que la aproximación es de un $92 \%$.

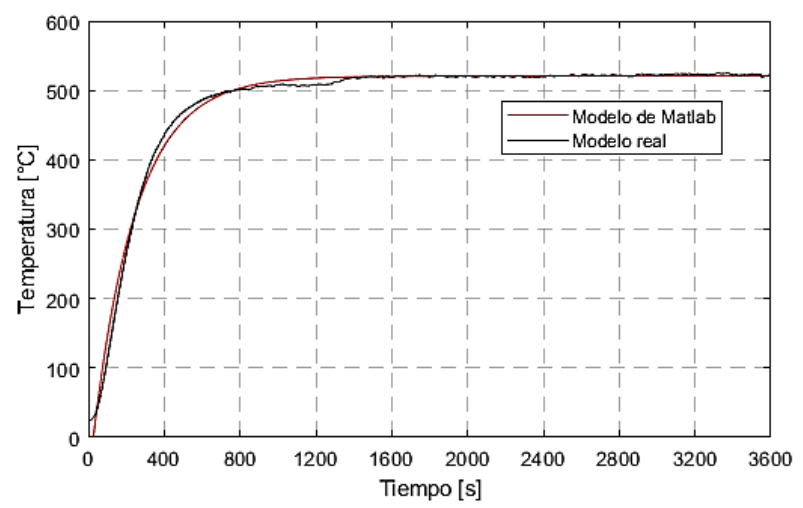

Figura 5 Graficas de la función de transferencia del modelo real y simulado de la planta

Fuente: Autoría propia

A continuación, se sintonizo el controlador PID, es decir, se determinaron las constantes $\mathrm{Kp}$, Ti y $\mathrm{T}_{\mathrm{d}}$ del controlador, con herramienta "PID tuner" del software de Matlab $^{\circledast}$, utilizando el diagrama, ver figura 6, Donde $K_{P}=0.4767 T_{i}=.00371$ y $T_{d}=5.7580$.

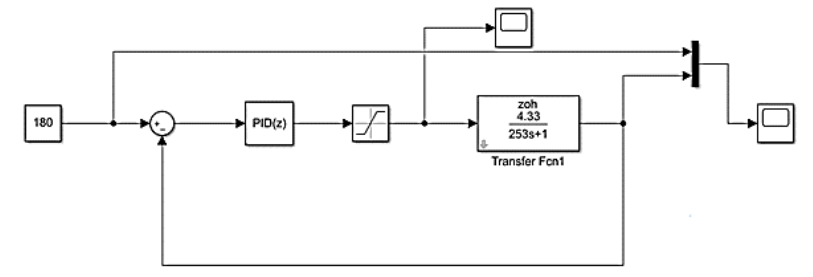

Figura 6 Diagrama en Simulink ${ }^{\circledR}$ para sintonización de controlador PID

Fuente: Autoría propia

Figura 7 , se muestra el diagrama del controlador propuesto para el sistema de control de temperatura en lazo cerrado del sistema de termoformado. Primeramente, se obtiene el error que es la diferencia entre la entrada de referencia y la señal de retroalimentación que proviene del termocuplas tipo k. Esta señal de error es enviada al controlador PID realizando las correcciones necesarias en la potencia que se suministra a la resistencia. Cuando el error es igual a cero no realiza una ninguna corrección y en caso contrario el PID genera una señal y se envía al TM1 que genera un voltaje digital en la salida de la tarjeta de desarrollo.

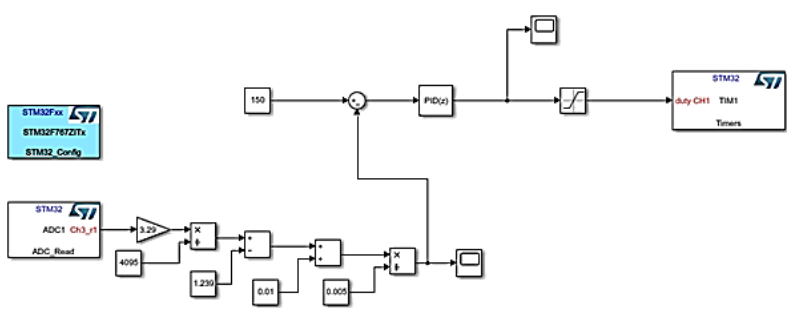

Figura 7 Programa del controlador PID en Simulink ${ }^{\circledR}$. Fuente: Autoría propia

La señal eléctrica que suministra el microcontrolador es digital, el circuito de control de fase utiliza una señal analógica, en la figura 8 , se muestra el circuito para acoplarlos y realizar la conversión de la señal digital analógico.

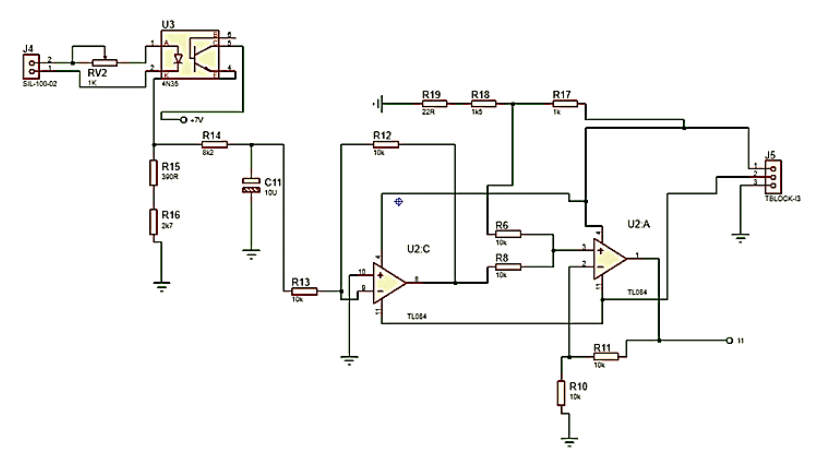

Figura 8 Circuito de aislamiento y acoplamiento entre el controlador y circuito de control de fase.

Fuente: Autoría propia 
Para controlar la potencia de la planta, se implementó el circuito de control de fase (TCA785), es decir, dependiendo del nivel de voltaje que suministre el controlador al circuito de control de fase entrega dos pulsos uno para el intervalo $0^{\circ} \mathrm{C}$ a $180^{\circ} \mathrm{C}$ y de $180^{\circ} \mathrm{C}$ a $360^{\circ} \mathrm{C}$, se muestra en la figura 9 , después los dos pulso de salida del circuito de control de fase son enviados al optotriac para aislar la señal de disparo y la compuerta del triac; por lo tanto, se regula la potencia de la planta dependiendo del ángulo de disparo, en el semiciclo positivo y negativo de la señal de corriente alterna, ver figura 10 .

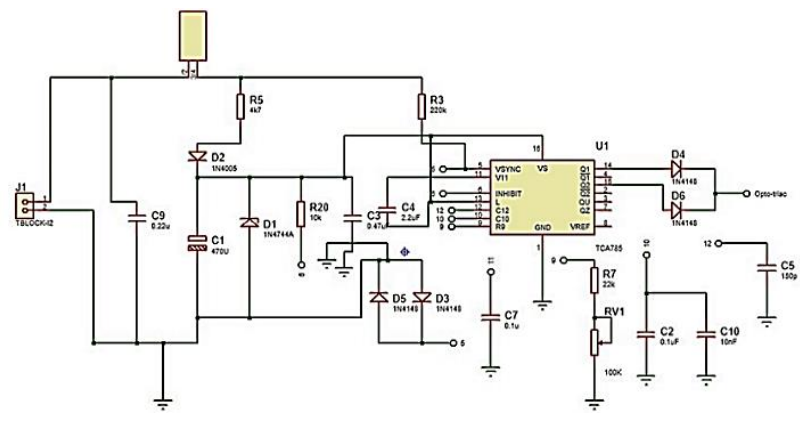

Figura 9 Circuito de control de fase Fuente: (Group, s.f.)

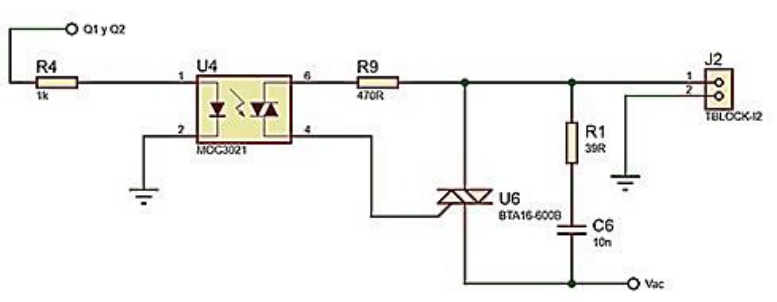

Figura 10 Circuito regulador de potencia de corriente alterna a la planta

Fuente: Autoría propia

La simulación del sistema de control PID, se muestra en la figura 11, donde el point es $180{ }^{\circ} \mathrm{C}$, el máximo sobre impulso $M_{P}=$ $3.02 \%$, el tiempo de establecimiento $T_{S}=$ 1150 segundo y el error $=0.1873^{\circ} \mathrm{C}$.

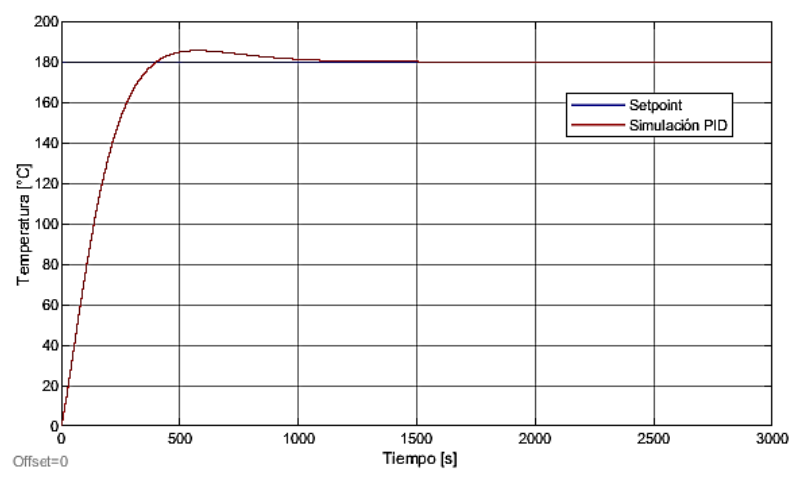

Figura 11 Curva característica del resultado de la simulación del controlador PID

Fuente: Autoría propia 


\section{Referencias}

Alfaro, V. (2005). Actualización del método de sintonización de controladores de Ziegler y Nichols. Ingeniería, 39-52.

Astróm, K., \& Hagglund, T. (1995). PID controllers: Theory, design and tuning. USA: Instr. Soc. Of America.

Grassi, E., \& Tsakalis, K. (2000). PID controller tuning by frequency loop-shaping application to diffusion furnace temperature control. IEEE Transactions on Control Systems Technology, 842-847.

Group, S. (s.f.). Obtenido de http://www.farnell.com/datasheets/1836360.pdf Güney, A., Temizkan, M., Tekin, S., Samuk, D., \& Cakir, O. (2020). Temperature Control of an Electric Furnace with Intuitive Control Methods. Science Literature.

Gutiérrez , M., \& Iturralde, S. (2017). Fundamentos Básicos de Instrumentación y control. Ecuador: UPSE.

Kuo, B. (1996). Sistemas de control autmático. Nueva York: Prentice Hall Hispanoamericana S. A.

Ogata, K. (1998). Ingeniería de control moderna. Prentice Hall.

Rosales, J., Enríquez , M. d., López, R., \& Mastache , J. E. (2019). Sistema de control de temperatura on-off implementado en el proceso de termoformado para elaboración de bloque a partir de envases multicapas. Revista de Ingeniería Eléctrica, 17-21.

st. (s.f.). Obtenido de https://pdf1.alldatasheet.es/datasheetpdf/view/933989/STMICROELECTRONICS/S TM32F767ZI.html

Vargas , B. (2012). Efectos del retardo en el control de lazo cerrado de plantas sobreamortiguadas. Industrial Data, 110-119.

Yanmei, W., Yanzhu, Z., \& Baoyu, W. (2011). The control research of PID in heating-furnace system. International Conference on Business Management and Electronic Information. 